\title{
COMPARATIVE STUDY OF STREPTOCOCCAL PHARYNGITIS IN NABEUL: CLINICAL JUDGMENT VERSUS RAPID ANTIGEN TEST VERSUS NEAPOLIS SCORE ASSOCIATED WITH RAPID ANTIGEN TEST
}

\author{
H. Bachrouche ${ }^{1}$, K. Chabbouh ${ }^{1}$, K. Chraiet ${ }^{1}$, A. Guedria ${ }^{1}$, H. Smaoui ${ }^{2}$, K. Aissa ${ }^{1}$ \\ ${ }^{1}$ El Manar University - University of Medecine of Tunis - \\ Mohamed Tlatli Hospital, Pediatrics and Neonatology, Nabeul, Tunisia \\ ${ }^{2}$ El Manar University - University of Medecine of Tunis - \\ Bechir Hamza children's Hospital, Bacteriology, Tunis, Tunisia
}

\section{Background}

$>$ Pharyngitis is the cause of many pediatric consultations.

$>$ Streptococcal pharyngitis (SP), which justifies antibiotics' prescription, accounts for only 15 to $30 \%$ of cases.

$>$ Examination of the throat does not distinguish between SP and viral pharyngitis.

$>$ Currently, there is no international consensus on the management of pharyngitis in children.

Aim:

to compare three strategies of management of pharyngitis in terms of efficiency and economic profitability by taking the throat culture as a reference.

Methods: (chart 1)

$>$ We carried out a prospective study over a period of 6 months on children aged from 2 to 15 years diagnosed with pharyngitis.

$>$ We divided the 180 children included into 3 groups of 60 each representing the $\mathbf{3}$ strategies:

1. strategy based on clinical judgment only,

2. strategy based on rapid antigen test (RAT) only

3. strategy based on the Neapolis score associated with RAT.

\begin{tabular}{lc}
\multicolumn{2}{c}{ Neapolis score } \\
\hline \multicolumn{1}{c}{ criteria } & Points \\
\hline Fever $>38{ }^{\circ} \mathrm{C}$ & +1 \\
Absence of Cough & +1 \\
Painful cervical nodes & +1 \\
Tonsillitis (swelling or exudate) & +1 \\
\hline
\end{tabular}

Results:

$>$ The prevalence of SP was $18.9 \%$ in our study.

$>$ A relationship was established between the SP and difficulty swallowing, absence of cough, exudate tonsillar and enlarged cervical nodes.

$>$ Multivariate analysis showed that only the absence of cough had an independent discriminatory value.

$>$ The sensitivity and specificity of the first strategy were $\mathbf{8 7 . 5 \%}$ and $11.5 \%$, respectively. Those of the second strategy were $\mathbf{8 3 . 3} \%$ and $\mathbf{9 7 . 9 \%}$. Those of the 3rd strategy were $\mathbf{8 5 . 7 \%}$ and 89.1\%. (Table 1, $2,3,4$ ).

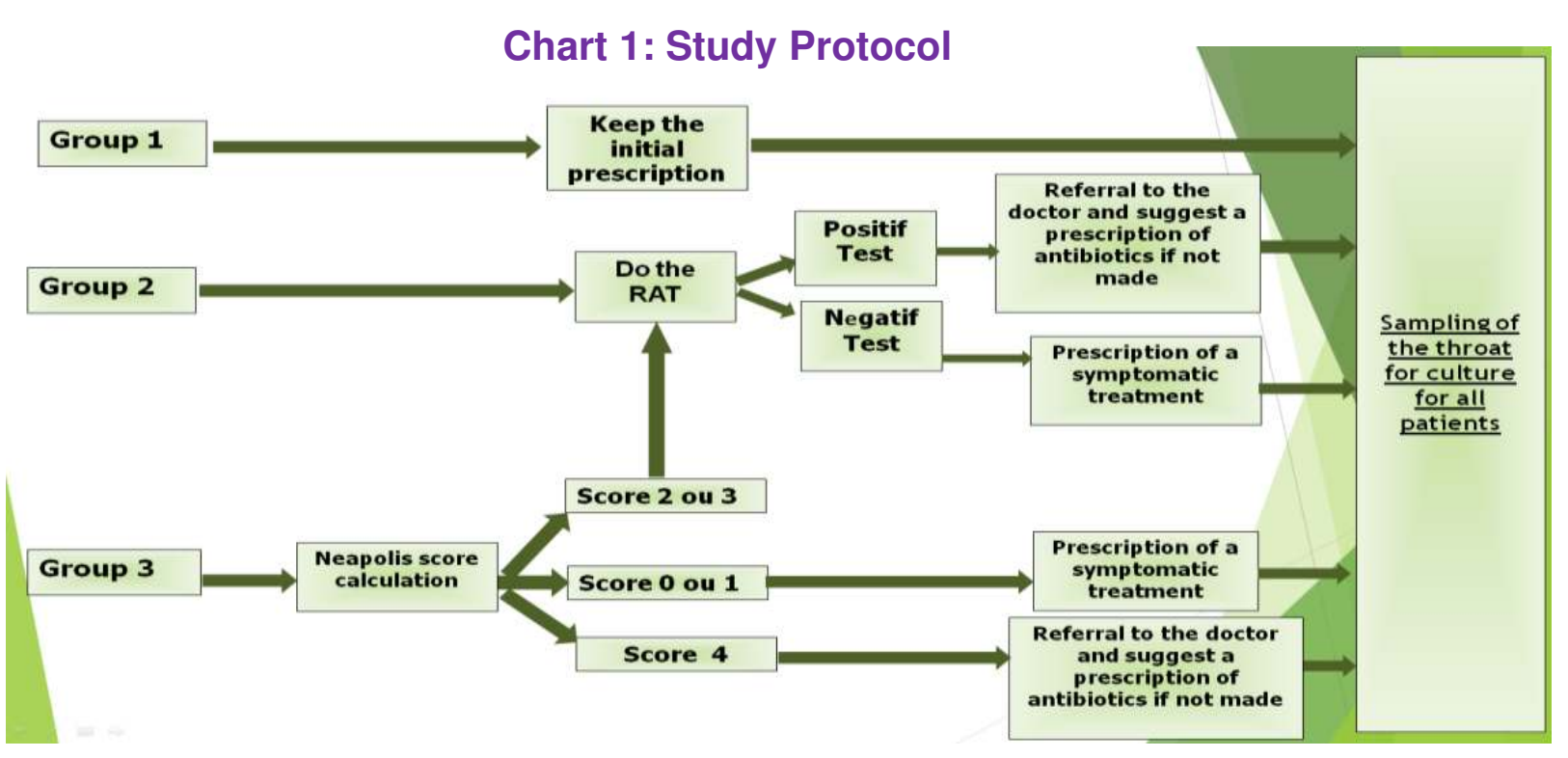

$>$ The 1st and 2nd strategies were the most expensive with a total cost of 344 Dinars and 337.8 Dinars respectively.

$>$ The total cost of the $3^{\text {rd }}$ strategy was 287 Dinars (1Euro=2,6Dinars).

$>$ All cultured Streptococus strains were completely sensitive to betalactams and macrolides, and resistant to tetracyclines in $50 \%$ of cases.

\section{Comments:}

$>$ The clinical examination does not allow us to distinguish between streptococcal and non-streptococcal pharyngitis.

$>$ It leads to overconsumption of antibiotics and leads to an increase in the rate of resistant germs.

$>$ The strategy based on RAT alone and that based on RAT combined with Neapolis score are the most effective, but the 3rd strategy remains the most economical.

Conclusion:

$>$ In light of our work, we recommend adopting the third management strategy (RAT associated with Neapolis score) as it has the best cost-effectiveness.

$>$ We then propose to integrate it into a international protocol for the management of children's pharyngitis.

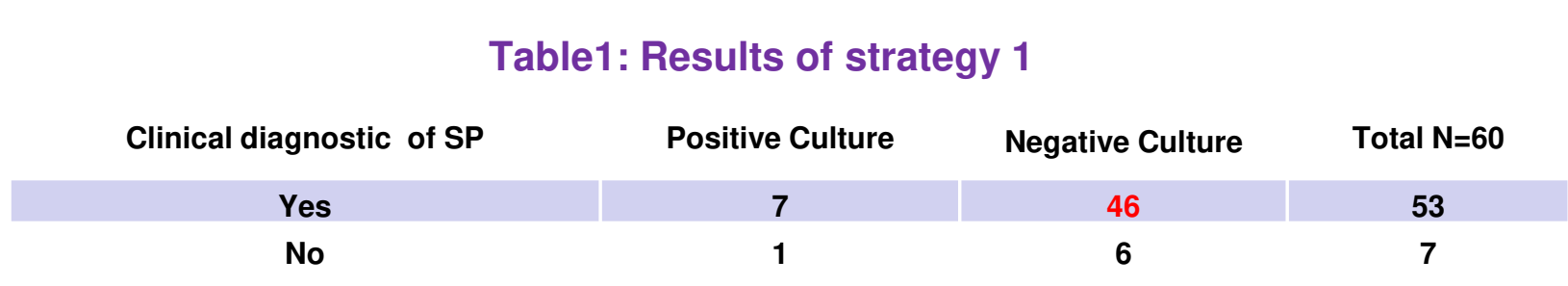

Table2: Results of strategy 2

\begin{tabular}{cccc}
\hline RAT Resultat & Positive Culture & Negative Culture & Total $\mathrm{N}=60$ \\
\hline Positif & 10 & 1 & 11 \\
\hline Negatif & 2 & 47 & 49
\end{tabular}

Table 3: Results of strategy 3

\begin{tabular}{ccccc} 
NeapolisScore & RAT & Positive Culture & Negative Culture & Total N=60 \\
Between 0 and 1 & Not done & 0 & 9 & 9 \\
\multirow{2}{*}{ Between 2 et 3} & Positif & 10 & 2 & 12 \\
& Negatif & 2 & 32 & 34 \\
More than 4 & Not done & 2 & 3 & 5
\end{tabular}

Table 4: Efficiency comparaison strategies

\begin{tabular}{|c|c|c|c|c|}
\hline Groups & Sensibility $\%$ & Specificity $\%$ & PPV $\%$ & PNV \% \\
& $($ IC) & $($ IC) & (IC) & (IC) \\
\hline Group 1 & $87,5 \%$ & $11,5 \%$ & $13,2 \%$ & $85,7 \%$ \\
& $(46,7 \%-99,3 \%)$ & $(4,8 \%-24,1 \%)$ & $(57,1 \%-99,5 \%)$ & $(42 \%-99,2 \%)$ \\
\hline \multirow{2}{*}{ Group 2 } & $83,3 \%$ & $97,9 \%$ & $90,9 \%$ & $95,9 \%$ \\
& $(50,9 \%-97,1 \%)$ & $(87,5 \%-99 \%)$ & $(57,1 \%-99,5 \%)$ & $(84,9 \%-99,3 \%)$ \\
\hline \multirow{2}{*}{ Group 3 } & $85,7 \%$ & $89,1 \%$ & $70,6 \%$ & $95,3 \%$ \\
& $(56,2 \%-97,5 \%)$ & $(75,-\%-95,9 \%)$ & $(44 \%-88,6 \%)$ & $(82,9 \%-99,2 \%)$ \\
\hline
\end{tabular}

References

, Kaplan EL, Schwartz RH. Practice Management of Group A Streptoco

Cohen J, Levy C, Chalumeau M, Bidet P, Cohen R. Rapid antigen detection tests for group A streptococcus in children with

pharyngitis. Arch Pediatr. 2014;21 Suppl 2:S78-83.

validity of a sore throat score in family practice. CMAJ. 2000;163(7):811-5.
Author Names and Contact Details:

Dr Haythem Bachrouche email:

haythem.bachrouche@gmail. 